\title{
Detection and Extinguishing Forest Fires using Wireless Sensor and Actor Networks
}

\author{
M.P.Sivaram Kumar \\ Assistant Professor \\ Department of CSE \\ DMl college of Engineering \\ Palanchoor, Chennai-123.
}

\author{
S.Rajasekaran \\ Professor \\ Department of Mathematics \\ B S Abdur Rahman University \\ Vandalur, Chennai-48
}

\begin{abstract}
Forests are rich containment of resources and they play a vital role in preserving and maintaining the environment. The major hazard of the forests is forest fire as the consequence of the forest fire is catastrophic in nature. Hence it is of great importance that forest fire occurrence must be detected and extinguished before the fire spreads and destroys the resources. A lot of detection mechanisms are available for forest fire in the literature using wireless sensor networks and other methods. In this paper we propose a quadrant based clustering and deterministic deployment of nodes for detecting and extinguishing forest fires using Wireless Sensor and Actor Networks (WSANs).
\end{abstract}

\section{General Terms}

Forest fires, Wireless Sensor and Actor Networks

\section{Keywords}

Deterministic deployment, Quadrant based Clustering

\section{INTRODUCTION}

The forest is considered to be the one of the most indispensable resources and the fatal threat to forests is forest fires. It is widely reported that a total of 77,534 wildfires burned 6,790,692 acres in USA during 2004 [1]. Hence it is necessary to carry out research activities to detect and extinguish fires to preserve valuable resources and environment. The best method to detect $h$ fires is by fire modeling because we can understand and predict possible fire behavior without getting burned [2]. In olden days fire detection is carried out by visual inspection of large areas with coverage radius of up to $20 \mathrm{~km}$ and daily walk through predefined paths during fire season by fire department personnel [3]. In the literature most of the forest fire detection mechanisms are based on satellite images [4].But the long scan period and low resolution of satellite images reduces the effectiveness of satellite based forest fire detection mechanisms. In order to overcome the difficulties associated with satellite image based detection mechanisms many works have been reported in the literature using wireless sensor networks $[5,6]$. But most of the works are concerned only with detection mechanisms and message will be sent to sink / gateway/internet and actions will be taken separately. In [7] a cluster based wireless sensor network for forest fires was presented. Recent advances in technology have lead to emergence of distributed wireless sensor and actor networks which are capable of observing the physical world, processing the data, making decisions based on the observations and perform appropriate actions. WSANs are very much used in Habitat and Environment monitoring [8]. In this paper we explore the use of WSANs technology in forest fire detection and extinguishing. The remainder of the paper is organized as follows: Section 2 describes the WSAN model for forest fire Detection and Extinguishing. In Section 3 we present how WSAN model is used in forest fire detection and extinguishing. Section 4 describes simulation results. Section 5 concludes the paper.

\section{WSAN MODEL FOR FOREST FIRES}

Wireless sensor and actor networks refer to a group of sensors and actors linked by wireless medium to perform distributed sensing and acting tasks. In WSANs the role of sensors is to gather information from the environment, while actors collect and process data and perform appropriate actions [8]. In Forest fire model of WSANs We have used two types of nodes(i) Beacon Nodes i.e., Nodes know their Location information (ii) cluster head Nodes to which all Beacon nodes are clustered based on quadrant and one actor.

\subsection{Model assumptions}

1. Forest Area is divided into $\mathrm{n} \times \mathrm{n}$ square cells also called grids [9].

2. The sensor node is placed at the center of the each grid.

3 . The sensing range of node is ' $r$ ' and side of square cell is ' $D$ ' and $2 \mathrm{r}=\mathrm{D}[\mathbf{9}]$.

4 The nodes are deployed deterministically and are static [10].

5. Nodes belonging to a quadrant are clustered to Cluster Head $(\mathrm{CH})$ which is placed at the corner of that Quadrant.

6. Actors contain fire extinguishing agent and are mobile.

7. Actor is available at the centre of domain facing towards East and returns to same place after it extinguishes fire.

8. Forest fire Propagates in all directions at same speed.

9. Since Propagation of fire is uniform and we are considering Surface fires only, fire originated in a region/grid $\mathrm{i}$ is detected by sensor i first [3].

10 The Quadrant based model is shown in Figure 1. 


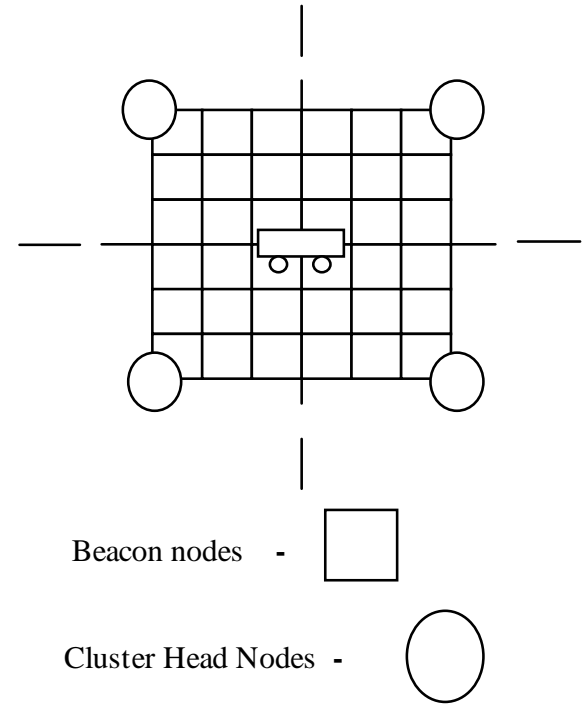

Actor Node -

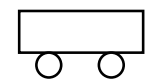

Figure 1 Quadrant based model

\section{FOREST FIRE DETECTION AND EXTINGUISHING}

Wireless Sensor and Actor Networks are used for forest fire detection and extinguishing system. The major issues to be considered in implementation are: (i) Sensor Deployment \& coverage (ii) Path planning for the actor (iii) Communication between beacon nodes, cluster head and actor

\subsection{Sensor deployment \& coverage}

A static network must be deployed according to a predefined shape in order to achieve Deterministic coverage and grid based deployment can be used for deterministic coverage [11]. In grid based scheme sensor deployment can be done in two methods:

a. Sensors can be placed at the centre of the grid [9]

b. Sensors can be placed at the intersection of the grid points [11]. In this work we have placed the sensors at the center of the grid because the number of sensors required for deployment is less.

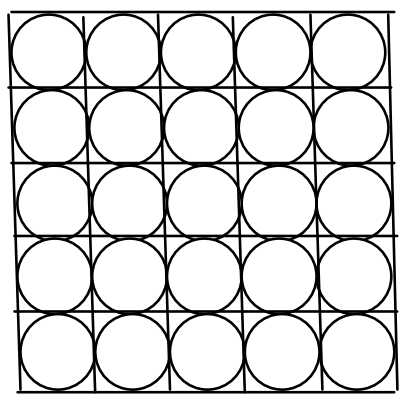

Figure 2 Sensors Placed at the center of grid

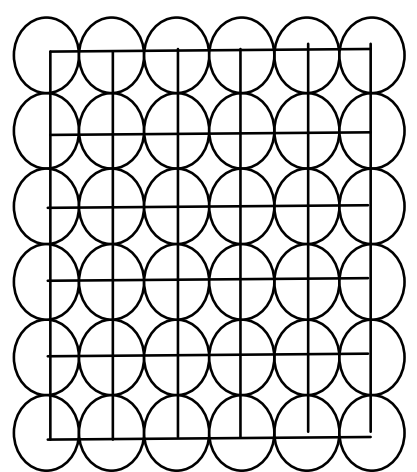

Figure 3 Sensors Placed at the intersection of grid points

Lemma 1: The number of sensors required for placing sensors at the center of the grid is less than placing sensors at the intersection of grid points.

From the above figures it is clear that for domain divided into $\mathrm{n} \times \mathrm{n}$ grids we can conclude

a. For Sensors placed at the center of grids: $n^{2}$ sensors will be required.

b. Sensors placed at the intersection of grid points: $n^{2}+2 n+1$ sensor will be required

Hence Lemmal is proved and sensors placed at the centre of grids require $(2 n+1)$ sensors lesser than sensors placed at the intersection of grid points. In case of grid based deployment problem of coverage of sensor field reduces to the problem of coverage of one cell and its neighbor because of symmetry [11]. Therefore if we take one square grid and calculate the uncovered Area it is approximately equal to $0.86 r^{2}$ [12] where $r$ is the sensing range. Since in this work we have assumed that fire starts at a point and if it occurs inside the uncovered area then it will be first detected by sensor in that region than any other sensor because the surface fire spreads at constant rate in all directions

\subsection{Path planning for the actor}

Path planning for mobile robots is defined as the search path in which the robot has to move in the prescribed environment [13]. The path planning of robot depending upon the environment is classified into a. static path planning and b. Dynamic path planning. The static path planning refers to environment where there are no moving objects and no Obstacles other than navigating object while dynamic path planning refers to environment where all are moving including Obstacles.

Depending on algorithm the path planning is local or global. Local path planning refers to robot which has no information about the environment and global path planning refers to robot that has information about the environment before it moves towards the target. In this work we have simulated the concepts and activities of a robot to an actor.

The distance which the actor has to move is calculated using law of cosines formula [14]

$d=$ square root $\left(b^{2}+c^{2}-2 b c \cos \alpha\right)$

Since we are using square grids $\alpha$ will always have 90 degrees and equation (1) reduces to

$\mathrm{d}=$ square root $\left(\mathrm{b}^{2}+\mathrm{c}^{2}\right) \quad$ (2) $\quad$ since $\cos 90=0$. 
The angle which the actor has to make in order to reach the target area is given by $\theta=\arctan (y / x)$

\subsection{Communication between beacon nodes, cluster head and actor}

WSANs is considered as a distributed control system which requires timely reaction based on the information received from sensor with an appropriate action .Hence Real time communication and coordination is of great importance in the context of WSANs [15]. In this work we classified the communication into (i) Beacon sensor to cluster head communication (ii) cluster head to actor communication

\subsection{Methodology}

The forest fire detection system consists of nodes deployed deterministically in a forest area and all the nodes know their location based on quadrants. Each node is equipped with a temperature sensor. Nodes continuously monitor the environment to check if there is fire or not in that grid. When change in temperature is detected they send packets which contain location measurements to Cluster Heads.

Since the focus of the paper is mainly on forest fire detection and extinguishing we assume that all report messages sent from the cluster members can reach their cluster heads successfully. Therefore packets sent by cluster members are received by the Corresponding $\mathrm{CH}$ Node. The $\mathrm{CH}$ Node then processes the packet and determines the quadrant from the location information. It then sends packets to the Actor.

The Actor in turn will process the packet to determine the distance and angle to be used in reaching the target area and to extinguish forest fire. The Packet format used by Beacon node, Cluster Head node is shown below in Figure 4 and Figure 5 respectively. The Algorithm used for quadrant calculation, Distance Estimation and Angle Calculation are shown in Algorithm 1, Algorithm 2 and Algorithm 3 respectively.

We have implemented the concept for single Actor and with slight modification we have implemented the concept for Multiple Actors keeping in mind the domain is very large.

\begin{tabular}{|c|c|c|}
\hline $\mathrm{XC}$ & $\mathrm{YC}$ & $\mathrm{CHNO}$ \\
\hline
\end{tabular}

Figure 4 Beacon Node Packet Format

Where

$\mathrm{XC}-\mathrm{x}$ Coordinate,

$\mathrm{YC}-\mathrm{y}$ Coordinate,

CHNO - Cluster Head Number to which packet is to be sent.

\begin{tabular}{|l|l|l|l|}
\hline $\mathrm{XC}$ & YC & QNO & AA \\
\hline
\end{tabular}

Figure 5 Cluster Head Node Packet Format

Where

$\mathrm{XC}-\mathrm{x}$ Coordinate,

$\mathrm{YC}-\mathrm{y}$ Coordinate,

QNO - Quadrant Number,

AA - Address of the Actor to which the Packet is to be sent.

\section{Algorithm 1: Quadrant Calculation}

Step 1: Read the packet sent by beacon node to determine $\mathrm{X}$-coordinate and $\mathrm{y}$-coordinate values

Step2: Check the values of XC and YC

2.1 if $\mathrm{XC}$ is Positive and $\mathrm{YC}$ is Positive then

Fire is in first Quadrant i.e. $\mathrm{QNO}=1$

2.2 if $\mathrm{XC}$ is Negative and YC is Positive then

Fire is in first Quadrant i.e. QNO=2

2.3 if $\mathrm{XC}$ is Negative and YC is Negative then

Fire is in first Quadrant i.e. QNO=3

2.4 if $\mathrm{XC}$ is Positive and $\mathrm{YC}$ is Negative then

Fire is in first Quadrant i.e. $\mathrm{QNO}=4$

Step3: Return QNO

\section{Algorithm 2: Distance Estimation}

Step 1: Read the packet sent by Cluster Head to get the $\mathrm{X}$-coordinate and $\mathrm{y}$-coordinate values

Step2: Calculate Distance using

2.1 add the square value of $\mathrm{XC}$ and $\mathrm{YC}$ and assign it to $\mathrm{k}$

2.2Take the square root of $\mathrm{k}$ and assign it to d

Step 3: The original distance the actor has to move is calculated as follows: $\mathrm{d}=\mathrm{d}-(\mathrm{D} / 2)$

Step4: Return d 


\section{Algorithm 3: Angle Calculation}

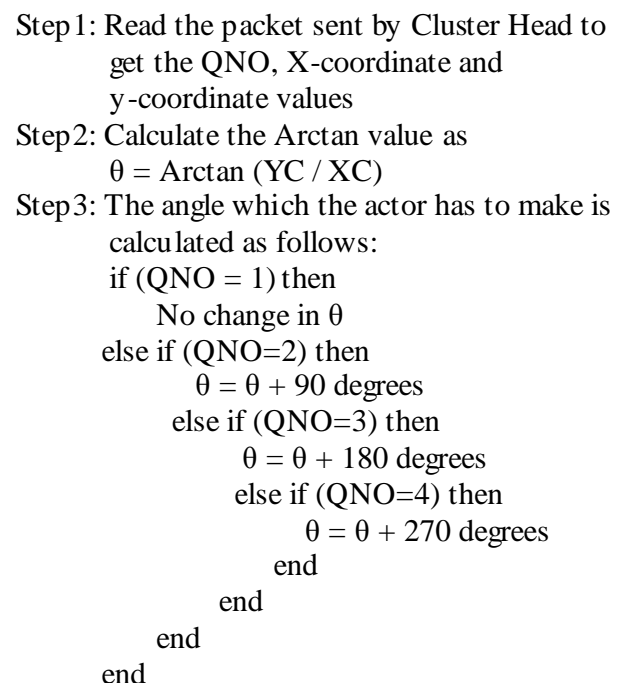

Step4: Return $\theta$

\section{SIMULATION AND RESULTS}

The Forest environment is simulated and run using Abode Flash Action script. The Test results are as expected. We have assumed the forest environment to contain only bush and herbs i.e. no obstacle so that actor can move freely with out any disturbances and it is applicable to savanna type forest. Moreover we have tested the Environment for both single actor and multiple actors. To test the effect of ignition point on the success rate of fire detection algorithm we have started the fire from different grids of the rectangular area and in all instances the fire is extinguished. The simulated results are shown below.

\subsection{Simulation of fire and Actor's Approach}

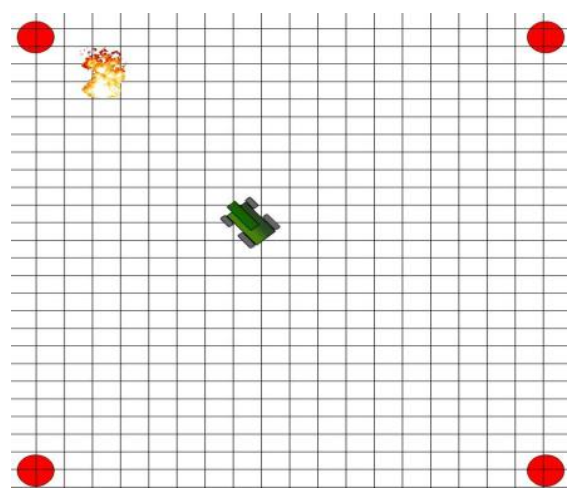

\subsection{ACTOR Extinguishing Fire}

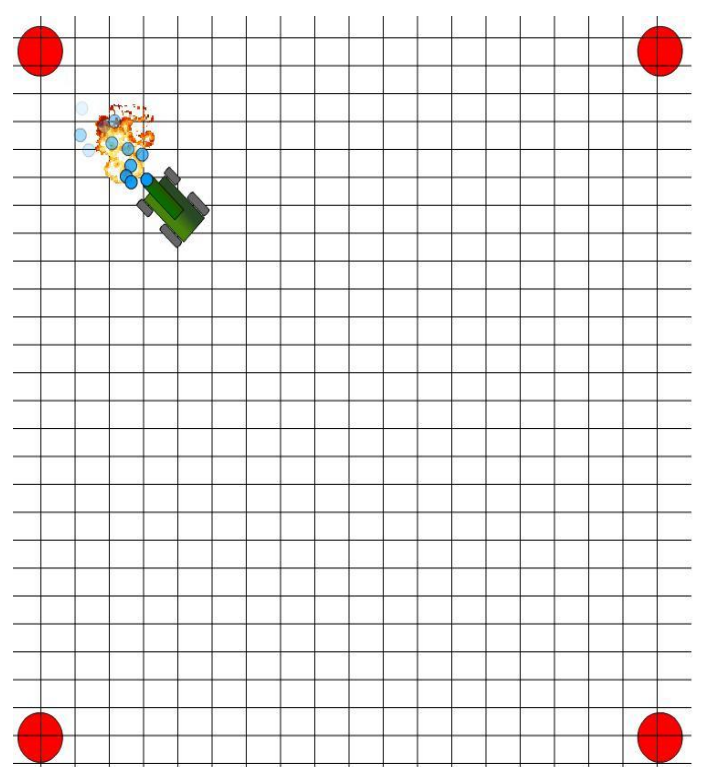

\subsection{Multiple actors in the test environment}

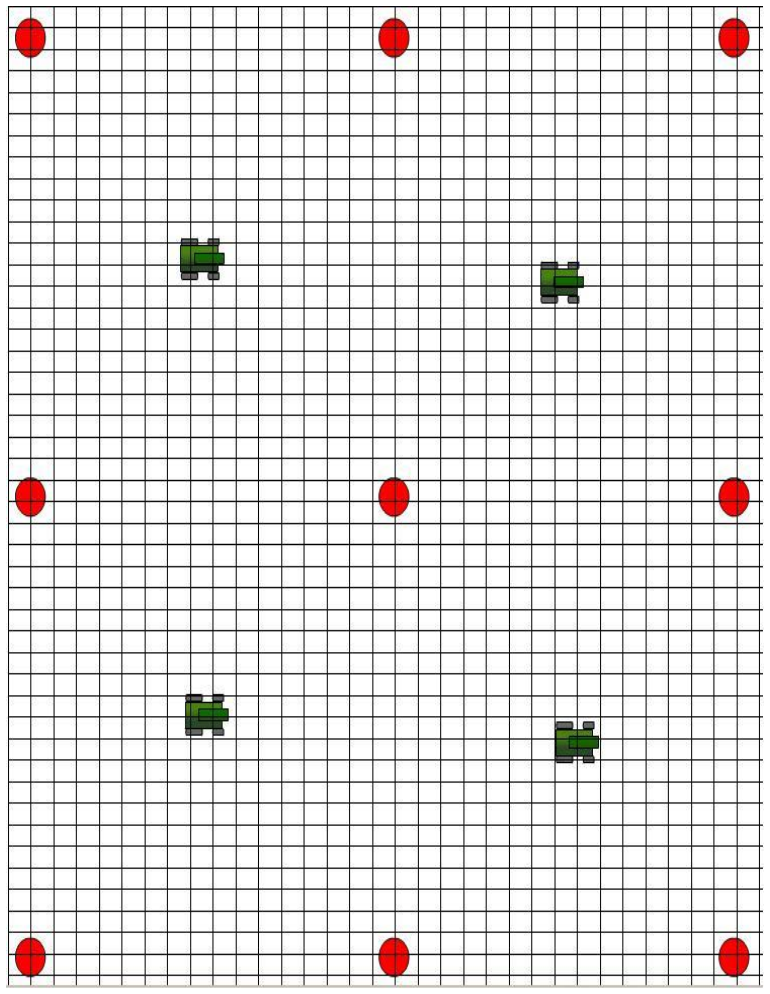




\subsection{Multiple actors extinguishing fires}

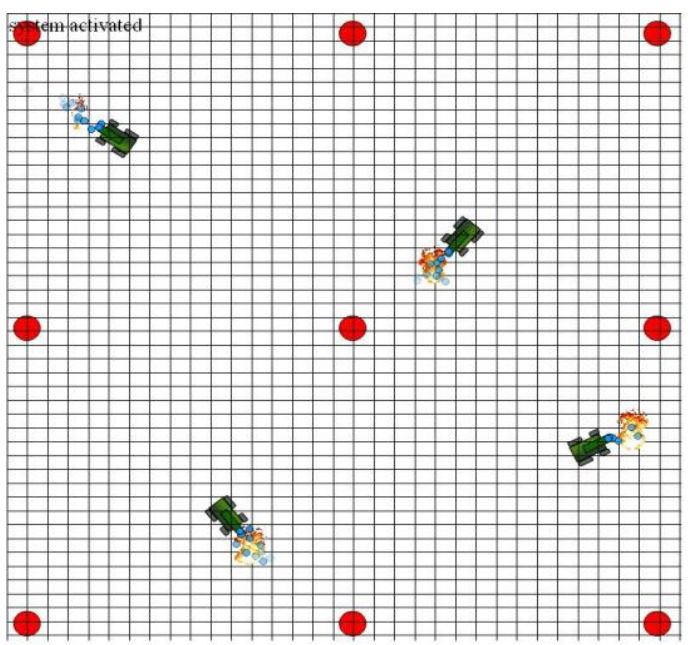

\section{CONCLUSION AND FUTURE ENHANCEMENTS}

In this paper we have presented the design and implementation of forest fire detection and extinguishing system using Wireless sensor and actor networks. The nodes are deployed deterministically and they are assumed to know the location based on quadrants. The Actor moved to the target area on the assumption that there are no obstacles. In future we will try to incorporate some Obstacles and random deploy ment of nodes.

Further the issues to be considered in future are (i) power back up for nodes (ii) how to refill exhausted extinguishing agent and (iii) local path planning of actors (iv) location calculation in random deployment of nodes.

\section{REFERENCES}

[1] http://www.nifc.gov/fireinfo/2004/index.html,"Wildland fire season 2004: statistics and summaries," National interagency coordination center

[2] Qasim Siddque ,"Survey of Forest fire simulation", Global journal of computer science and technology pp. 137-148

[3] Pablo.I.Fierens ," Number of Wireless sensors needed to detect a wildfire",Informal Publication

[4] MODIS Web available at: http://modis.gsfc.nasa.gov/

[5] Nukhet Sazak and Haldun Abdullah ," The importance of using Wireless Sensor Networks for Forest Fire Sensing and detection in Turkey, 5th International Advanced Technologies Symposium (IATS'09), May 13-15, 2009, Karabuk, Turkey

[6] B.Kosucu,K.Irgan,G.Kucuk,S.Bay dere," FireSenseTB: A wireless sensor Networks TestBed for Forest fire Detection pp 1173-1177

[7] Junguo Zhang,Wenbin LI,Zhongxing YIN, Shengbo LIU,Xiaolin GUO, "Forest Fire Detection System Based on wireless sensor Network",pp 520-523 ICIEA2009
[8] M.Hefeeda and M.Bagheri,"Wireless sensor networks for early detection of forest fires,"International workshop on Mobile Adhoc and sensor systems for global and homeland security (MASS-GHS'07), in conjunction with IEEE MASS'07, Pisa, Italy, October2007

[9]BenyuanLiu,,DonTowsley,"On the coverage and detectability of large scale wireless sensor networks" Proceedings of the workshop on Modeling and Optimisation in Mobile Adhoc and wireless networks (WiOpr'03),Mar2003.

[10]Ian F.Akyildiz,Ismail H.Kasimoglu "Wireless Sensor and Actor Networks: research challenges", Mobile Adhoc networks vol.no 2 pp.no 351-367

[11]S.Meguerdichian,F.Koushanfar,M.Potkonaj, M.B.Srivastava "Coverage problems in wireless adhoc sensor networks,"in proc.IEEE Infocom,2001,pp.1380-1387

[12] Jiehuichen,M.B.Salim \& M.Matsumoto “A single mobile target tracking in voronoi based clustered wireless sensor network" Journal of Information processing systems vo1.7,March 2011,pp.no 17-28.

[13] Buniyamin N,Wan Ngah w.A.J.,sariff N.,Mohammad z,"A Simple Local Path Planning Algorithm for Autonomous Mobile robots", International journal of systems applications, Engineering \& development issue 2,volume 6 pp 151-159,2011

[14] Trigonometry available at: http:// en.wikipedia.org/wikipedia/

[15] T. Melodia, D. Pompili,V. C. Gungor, and I. F. Akyildiz, "Communication and coordination in wireless sensor and actor networks," IEEE Transactions on Mobile Computing, vol. 6, no. 10, pp. 1116-1129, Oct. 2007. 